\title{
Efeito de 16 semanas de treinamento com pesos sobre a pressão arterial em mulheres normotensas e não-treinadas
}

\author{
Aline Mendes Gerage ${ }^{1,2}$, Edilson Serpeloni Cyrino ${ }^{1,2,3}$, Durcelina Schiavoni ${ }^{1}$, Fábio Yuzo Nakamura ${ }^{1,2}$ \\ Enio Ricardo Vaz Ronque ${ }^{1,3}$, André Luiz Demantova Gurjão ${ }^{1,4}$ e Sebastião Gobbi ${ }^{4}$
}

\section{RESUMO}

O propósito deste estudo foi analisar o impacto de 16 semanas de treinamento com pesos (TP) sobre a pressão arterial (PA) em mulheres normotensas. Para tanto, participaram do estudo 21 mulheres jovens $(20,5 \pm 2,1$ anos), não-treinadas, que foram separadas, aleatoriamente, em grupo treinamento $(G T=13)$ e grupo controle $(\mathrm{GC}=8)$. O protocolo de TP foi estruturado de forma progressiva, em duas etapas, com duração de oito semanas cada, sendo executado com freqüência semanal de três sessões. Medidas de PA foram realizadas em repouso por meio do método auscultatório nos diferentes momentos do estudo (linha de base, após oito e 16 semanas). O teste de Shapiro-Wilk foi utilizado para análise da normalidade dos dados. ANOVA two-way para medidas repetidas foi utilizada para as comparações entre os grupos ao longo do tempo, seguida pelo teste post-hoc de Scheffé quando $P<$ 0,05 . Uma interação grupo vs. momento foi encontrada nos valores de PA sistólica, diastólica e média, com reduções significantes, ao longo das 16 semanas de acompanhamento, na ordem de 5 , 8 e $6 \mathrm{mmHg}$, respectivamente, no GT. Os resultados sugerem que 16 semanas de TP podem provocar importantes reduções na PA em mulheres jovens, normotensas e, previamente, não-treinadas.

\section{ABSTRACT \\ Effect of 16-week weight training over blood pressure in normotensive and non-trained women}

The aim of this study was to analyze the impact of a 16-week weight training (WT) over blood pressure (BP) in normotensive women. The sample consisted of 21 young women $(20.5 \pm 2.1$ years), non-trained, who were randomly grouped in training group ( $T G=13)$ and control group (CG = 8). The WT protocol was progressively structured, in two phases, with duration of eight weeks each, being performed with weekly frequency of three sessions. $B P$ measurements were conducted at rest through the auscultatory method, in the different moments of the study (baseline, af-

1. Grupo de Estudo e Pesquisa em Metabolismo, Nutrição e Exercício, Centro de Educação Física e Esporte, Universidade Estadual de Londrina.

2. Grupo de Estudo das Adaptações Fisiológicas ao Treinamento, Centro de Educação Física e Esporte, Universidade Estadual de Londrina.

3. Grupo de Estudo e Pesquisa em Atividade Física e Exercício, Centro de Educação Física e Esporte, Universidade Estadual de Londrina.

4. Laboratório de Atividade Física e Envelhecimento, Departamento de Educação Física, Universidade Estadual Paulista - Rio Claro.

Aceito em 3/7/07.

Endereço para correspondência: Edilson Serpeloni Cyrino, Grupo de Estudo e Pesquisa em Metabolismo, Nutrição e Exercício, Centro de Educação Física e Esporte, Universidade Estadual de Londrina, Rod. Celso Garcia Cid, km 380, Campus Universitário - 86051-990 - Londrina, PR, Brasil. E-mail: emcyrino@uel.br
Palavras-chave: Treinamento com pesos. Pressão arterial. Mulheres. Keywords: Weight training. Blood pressure. Women.

ter eight and 16 weeks). The Shapiro-Wilk test was used for analysis of the data normality. Two-way ANOVA for repeated measurements was used for the comparisons between groups during the time, followed by the Scheffé post-hoc test when $P<0.05$. A group $v$. moment interaction was found in the systolic, diastolic and mean BP values, with significant decreases, during the 16 weeks of follow-up, in the order of 5,8 and $6 \mathrm{mmHg}$, respectively, in the TG. The outcomes suggest that the 16 week-WT program may cause important reductions in BP in normotensive young women and previously non-trained.

\section{INTRODUÇÃO}

A prevalência da hipertensão arterial tem crescido acentuadamente em diversos países do mundo, atingindo tanto homens quanto mulheres, em diferentes faixas etárias ${ }^{(1)}$. Dentre as principais causas para o estabelecimento desse quadro destacam-se o reduzido nível de atividade física habitual e o excesso de gordura corporal, sobretudo em mulheres ${ }^{(2-4)}$. Assim, a adoção de estratégias de intervenção que incluam mudanças no estilo de vida pode favorecer a prevenção, bem como o tratamento dessa importante disfunção cardiovascular.

Nesse sentido, diversos estudos têm indicado que a prática de exercícios físicos regulares pode provocar modificações importantes na pressão arterial (PA), em indivíduos normotensos e hipertensos ${ }^{(5-11)}$.

Com base nas informações disponíveis na literatura, verifica-se que alguns pesquisadores advogam que programas de exercícios físicos predominantemente aeróbios seriam mais indicados do que os predominantemente anaeróbios para a redução da $P A^{(7,12)}$. Todavia, estudos de metanálise têm indicado que a prática de exercícios com pesos também pode contribuir para o tratamento e/ou prevenção de disfunções cardiovasculares, como a hipertensão

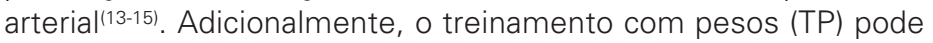
gerar modificações positivas em diferentes componentes da aptidão física, tais como: composição corporal, força, potência e resistência muscular.

Desse modo, a prática sistematizada de exercícios com pesos vem sendo encorajada por algumas das principais organizações internacionais(16-20), para a melhoria de diversos parâmetros relacionados à saúde.

Considerando que a PA é um importante indicador de saúde cardiovascular e que a prática de exercícios com pesos pode acarretar inúmeros benefícios, tanto para a saúde quanto para a qualidade de vida, acredita-se que o TP possa oferecer respostas fisiológicas e morfológicas benéficas a PA. Entretanto, verifica-se na literatura resultados bastante conflitantes associados às respostas pressóricas associadas ao TP, tanto de forma aguda quanto crônica. 
Vale ressaltar que grande parte das dificuldades em interpretar os resultados de vários estudos disponíveis na literatura envolvendo o TP e a PA, parece estar relacionada à falta de controle dos níveis de aptidão física prévia dos sujeitos investigados e aos protocolos experimentais adotados, que na maioria das vezes têm sido estruturados de forma muito diferenciada com relação ao volume (número de exercícios, séries e repetições, freqüência semanal) e à intensidade (carga, velocidade de execução dos movimentos, intervalos de recuperação entre as séries e os exercícios).

Assim, acredita-se que investigações que consigam controlar a maioria dessas variáveis, em indivíduos com níveis de aptidão física semelhantes, possam favorecer a compreensão da possível efetividade dos programas de TP sobre o comportamento da PA.

Portanto, o objetivo do presente estudo foi analisar o efeito de 16 semanas de TP sobre a pressão arterial sistólica (PAS), pressão arterial diastólica (PAD) e pressão arterial média (PAM) em mulheres jovens, normotensas e, previamente, não-treinadas.

\section{MÉTODOS}

\section{Sujeitos}

Trinta mulheres jovens, universitárias, na faixa etária de 18 a 26 anos, foram selecionadas voluntariamente para participar deste estudo e separadas, aleatoriamente, de forma balanceada, em dois grupos, treinamento (GT) e controle (GC). Como critérios iniciais de inclusão, as participantes deveriam ser sedentárias ou moderadamente ativas (atividade física regular inferior a duas vezes por semana), não terem participado regularmente de nenhum programa de TP ao longo dos últimos seis meses precedentes ao início do experimento e não apresentar quadro de hipertensão arterial ou pré-hipertensão. Além disso, cada participante respondeu, anteriormente ao início do estudo, um questionário sobre o histórico de saúde e nenhuma disfunção metabólica ou músculo-esquelética foi relatada.

Nove mulheres acabaram não concluindo o experimento por diversos motivos, incluindo abandono voluntário, não-cumprimento de procedimentos estabelecidos previamente ao início do estudo, problemas de saúde não relacionados ao protocolo experimental, ou problemas particulares. Assim, 21 mulheres compuseram a amostra analisada ( $\mathrm{GT}=13$ e $\mathrm{GC}=8$ ).

Todas as participantes, após receberem informações sobre as finalidades do estudo e os procedimentos aos quais seriam submetidas, assinaram um termo de consentimento livre e esclarecido. Vale ressaltar que este estudo faz parte de um projeto longitudinal que vem investigando o efeito do TP e da suplementação de creatina em mulheres. O estudo foi aprovado pelo Comitê de Ética em Pesquisa da Universidade Estadual de Londrina, de acordo com as normas da Resolução 196/96 do Conselho Nacional de Saúde sobre pesquisa envolvendo seres humanos.

\section{Antropometria}

Medidas antropométricas foram utilizadas para caracterização da amostra. A massa corporal foi mensurada em uma balança de leitura digital, da marca Urano, modelo PS 180A, com precisão de $0,1 \mathrm{~kg}$, ao passo que a estatura foi determinada em um estadiômetro de madeira com precisão de $0,1 \mathrm{~cm}$, de acordo com os procedimentos descritos por Gordon et al.(21). A partir dessas medidas foi calculado o índice de massa corporal (IMC), por meio da relação entre a massa corporal e o quadrado da estatura, sendo a massa corporal expressa em quilogramas $(\mathrm{kg})$ e a estatura em metros $(\mathrm{m})$.

\section{Pressão arterial}

Para a mensuração da PA foi empregado o método auscultatório, com auxílio de um esfigmomanômetro da marca Missouri e de um estetoscópio da marca BD.
Todas as medidas foram realizadas em situação de repouso, nos diferentes momentos do estudo: antes do início do experimento (M1), após oito (M2) e 16 (M3) semanas, por um único avaliador com experiência nesse tipo de medida. Em cada um dos momentos foram realizadas três medidas com intervalo de dois minutos entre elas, em três dias consecutivos, em horário semeIhante, no período da tarde. O valor médio entre as medidas obtidas nos diferentes dias foi registrado como valor de referência nos três momentos do estudo.

Para tanto, as participantes permaneceram sentadas em uma cadeira, em repouso, durante 10 minutos, antes do início da coleta de dados. As medidas foram obtidas sempre em condições semelhantes, no mesmo local e na mesma posição, com o braço direito sendo elevado até a altura do ponto médio do esterno e apoiado sobre uma mesa.

Vale ressaltar que as participantes foram orientadas previamente para que não realizassem nenhum tipo de atividade física vigorosa nas $24 \mathrm{~h}$ anteriores aos dias de coleta de dados e para que não estivessem em continência urinária no momento da realização das medidas de PA.

\section{Protocolo de treinamento com pesos}

O protocolo de TP foi realizado durante 16 semanas, em duas etapas, cada qual com a duração de oito semanas consecutivas, intercaladas por uma semana de intervalo, sem qualquer tipo de treinamento, para que fossem realizadas as reavaliações e reestruturações dos programas de treinamento.

O protocolo de treinamento nessas duas etapas teve como finalidade o processo de hipertrofia muscular e envolveu somente uma única programação de TP que foi executada em três sessões semanais, em dias alternados. A diferença entre essas etapas foi determinada pela forma de estruturação do protocolo de treinamento (escolha e ordenação dos exercícios), bem como pelo número total de exercícios executados, sendo utilizada uma montagem alternada por segmento, na primeira etapa, e uma montagem localizada por articulação, na etapa subseqüente. A adoção dessa estratégia tende a gerar uma sobrecarga progressiva, além de promover uma quebra da homeostase ao treinamento.

O programa de treinamento na primeira etapa foi composto por 10 exercícios, que foram executados na seguinte ordem: supino em banco horizontal, leg press $45^{\circ}$, puxada por trás do pescoço, mesa extensora, elevação lateral de ombros, mesa flexora, tríceps no pulley, panturrilha no leg press, rosca direta de bíceps e flexão abdominal.

Na segunda etapa, foram utilizados 12 exercícios, dispostos na seguinte ordem: supino em banco horizontal, crucifixo em banco inclinado, puxada por trás do pescoço, remada baixa completa, desenvolvimento por trás do pescoço, rosca direta de bíceps, rosca tríceps no banco horizontal, mesa extensora, leg press $45^{\circ}$, mesa flexora, panturrilha no leg press e flexão abdominal.

Vale ressaltar que, em ambas as etapas, foram empregados exercícios para os diferentes grupamentos musculares. Cada exercício foi executado em três séries de 8 a 12 repetições máximas $(R M)$, sendo adotado o método de cargas fixas. Nessas duas etapas, as únicas exceções foram os exercícios para os grupamentos musculares da panturrilha (15 a 20-RM) e do abdômen (30 a 50 repetições, sem sobrecarga adicional).

As cargas utilizadas foram compatíveis ao número de repetições máximas estipuladas para cada exercício. Os indivíduos receberam orientação para que as cargas de treinamento fossem reajustadas sempre que o número máximo de repetições preestabelecidas para cada exercício fosse atingido em todas as séries, na tentativa de que a intensidade inicial pudesse ser preservada.

Tanto as cargas iniciais quanto os reajustes periódicos, nas cargas utilizadas nos diferentes exercícios, foram estabelecidos com base nos resultados obtidos mediante a aplicação de testes de peso por repetições máximas ${ }^{(22)}$. Vale ressaltar que, em ambas as 
etapas, o intervalo de recuperação estabelecido entre as séries, em cada exercício, foi de 60 a 90s, e entre os exercícios de dois a três minutos.

As participantes foram orientadas, ainda, para que não realizassem nenhum outro tipo de atividade física regular sistematizada durante o período de duração do estudo, de modo que o impacto do TP pudesse ser avaliado de forma isolada.

\section{Tratamento estatístico}

O teste de Shapiro Wilk foi utilizado, inicialmente, para análise da distribuição dos dados. As características físicas iniciais dos dois grupos (GT e GC) foram comparadas pelo teste t de Student para amostras independentes com número de elementos diferentes. Análise de variância (ANOVA) $2 \times 3$ para medidas repetidas foi empregada para as comparações entre os grupos (GT e GC) e os momentos (M1, M2 e M3). O teste post hoc de Scheffé, para comparações múltiplas, foi empregado para a identificação das diferenças específicas nas variáveis em que os valores de F encontrados foram superiores ao critério de significância estatística estabelecido $(P<0,05)$.

\section{RESULTADOS}

As características físicas dos dois grupos (GT e GC) são apresentadas na tabela 1. Nenhuma diferença estatisticamente significante foi encontrada nas comparações entre os grupos no momento inicial do estudo.

TABELA 1

Características físicas iniciais dos grupos treinamento (GT) e controle (GC)

\begin{tabular}{lrrr}
\hline \multicolumn{1}{c}{ Variáveis } & GT (n= 13) & GC $(\mathbf{n}=\mathbf{8})$ & \multicolumn{1}{c}{} \\
Idade (anos) & $19,9 \pm 2,1$ & $21,1 \pm 2,2$ & 0,22 \\
Massa corporal $(\mathrm{kg})$ & $58,6 \pm 7,1$ & $57,2 \pm 8,5$ & 0,68 \\
Estatura $(\mathrm{cm})$ & $164,5 \pm 5,5$ & $164,1 \pm 7,8$ & 0,88 \\
IMC $\left(\mathrm{kg} / \mathrm{m}^{2}\right)$ & $21,6 \pm 2,1$ & $21,2 \pm 2,6$ & 0,70 \\
\hline
\end{tabular}

Nota: Os resultados estão expressos em valores médios ( \pm DP).

A tabela 2 apresenta informações relativas à PAS. Nenhuma diferença significante foi encontrada entre os grupos no M1 (linha de base). Entretanto, uma interação significante grupo vs. momento foi verificada ( $F=3,87 ; P<0,05)$, com redução significante da PAS no grupo GT entre M1-M2 $(5 \mathrm{mmHg})$, seguida de estabilização entre M2-M3. Por outro lado, nenhuma modificação foi identificada no $\mathrm{GC}(P>0,05)$.

\section{TABELA 2}

Comportamento da pressão arterial sistólica $(\mathrm{mmHg}) \mathrm{em}$ repouso, nos grupos treinamento (GT) e controle (GC), nos diferentes momentos do estudo

\begin{tabular}{ccclcc}
\hline Momentos & GT $(\mathbf{n}=\mathbf{1 3})$ & $\mathbf{G C}(\mathbf{n}=\mathbf{8})$ & Efeitos & $\mathbf{F}$ & $\boldsymbol{P}$ \\
M1 & $105 \pm 10$ & $105 \pm 10$ & Grupo & 0,60 & 0,45 \\
M2 & $100 \pm 9^{*}$ & $105 \pm 10$ & Momento & 2,66 & 0,08 \\
M3 & $100 \pm 9^{*}$ & $105 \pm 11$ & Grupo vs. Momento & 3,87 & 0,03 \\
\hline
\end{tabular}

* $P<0,05$ vs. M1

Nota: Os resultados estão expressos em valores médios ( $\pm \mathrm{DP})$. M1 = pré-treinamento; $\mathrm{M} 2=$ após oito semanas; $\mathrm{M} 3$ = após 16 semanas.

Com relação à $P A D$, ANOVA também identificou uma interação entre grupo vs. momento ( $F=6,73 ; P<0,01)$. Embora uma redução na PAD já tenha sido observada entre $\mathrm{M} 1-\mathrm{M} 2(5 \mathrm{mmHg})$ no GT, diferenças estatisticamente significantes só foram encontradas entre M1-M3 $(8 \mathrm{mmHg} ; P<0,05)$. Nenhuma modificação estatisticamente significante foi encontrada no GC entre M1-M3 $(P>0,05)$ (tabela 3).
TABELA 3

Comportamento da pressão arterial diastólica $(\mathrm{mmHg})$ em repouso, nos grupos treinamento (GT) e controle (GC), nos diferentes momentos do estudo

\begin{tabular}{ccclcr}
\hline Momentos & GT (n= 13) & GC (n= 8) & \multicolumn{1}{c}{ Efeitos } & F & $\boldsymbol{P}$ \\
M1 & $73 \pm 8$ & $69 \pm 9$ & Grupo & 0,10 & 0,75 \\
M2 & $68 \pm 6$ & $70 \pm 8$ & Momento & 4,25 & 0,02 \\
M3 & $65 \pm 7^{*}$ & $70 \pm 6$ & Grupo vs. Momento & 6,73 & $<0,01$ \\
\hline
\end{tabular}

* $P<0,05$ vs. M1

Nota: Os resultados estão expressos em valores médios ( \pm DP). M1 = pré-treinamento; $\mathrm{M} 2=$ após oito semanas; M3 = após 16 semanas.

No que se refere à PAM (figura 1), um decréscimo significante foi observado já no final das primeiras oito semanas de intervenção (M1-M2) no GT $(P<0,05)$. A partir daí, a redução verificada entre M2-M3 ocorreu de forma mais discreta e não significante ( $P$ $>0,05)$. Por outro lado, nenhuma modificação estatisticamente significante foi observada no GC entre M1-M3 $(P>0,05)$. Assim, uma interação grupo vs. momento foi identificada $(F=7,67 ; P<$ $0,05)$.

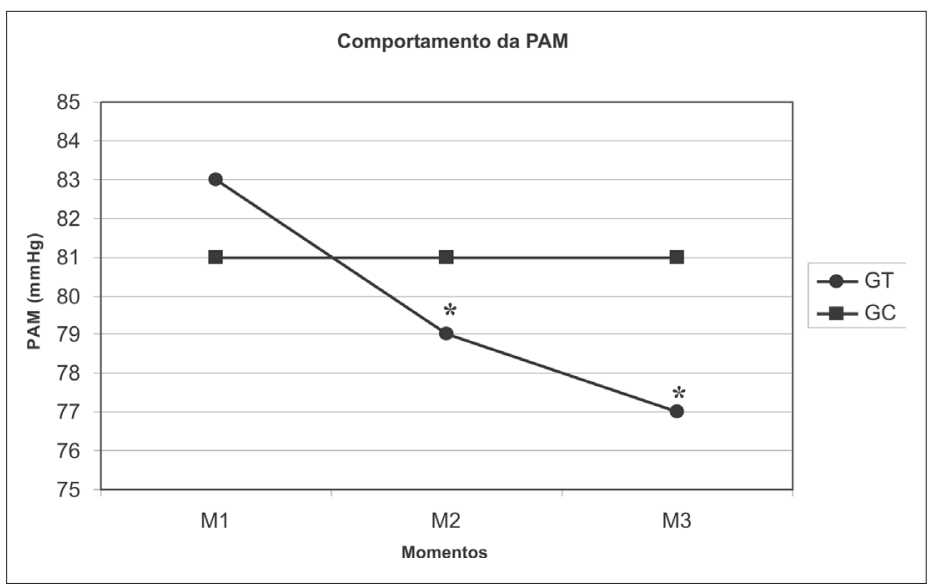

Figura 1 - Valores médios de pressão arterial média (PAM) nos diferentes momentos do estudo, nos grupos treinamento $(G T=13)$ e controle $(G C=$ 8)

${ }^{*} \mathrm{P}<0,05$ vs. $\mathrm{M} 1$; Interação grupo vs. momento $(\mathrm{F}=7,67 ; \mathrm{P}<0,05) ; \mathrm{M} 1=$ pré-treinamento; $M 2$ = após oito semanas; $M 3$ = após 16 semanas

\section{DISCUSSÃO}

Diversos estudos na literatura têm investigado o impacto de programas de exercícios físicos sobre a PA, de forma crônica, em diferentes populações, de ambos os sexos e em diversas faixas etárias. Considerando que a população masculina tem sido alvo da maioria desses estudos, bem como o modelo de treinamento preferido tem envolvido exercícios físicos predominantemente aeróbios, o presente estudo buscou, de forma específica, investigar o impacto de programas de TP sobre a PA em mulheres jovens, normotensas e, previamente, não-treinadas.

Por muito tempo acreditou-se que o TP pudesse provocar elevação crônica na PA em virtude de uma possível hipertrofia do músculo liso vascular, bem como de um possível aumento da resistência vascular periférica total(15). No entanto, nos últimos anos, com o crescimento do número de investigações na área, observase um aumento acentuado das informações relacionadas aos possíveis benefícios do TP para o controle da PA, tanto em indivíduos hipertensos quanto em normotensos ${ }^{(23-25)}$.

Recentemente, Rakobowchuk et al.(26) demonstraram que um remodelamento arterial periférico positivo pode ocorrer após a participação em programa de TP direcionado para hipertrofia muscular, tal qual o utilizado no presente estudo. De fato, um incre- 
mento significante no diâmetro médio da artéria braquial em repouso foi observado após seis semanas de TP. A partir daí, verificou-se estabilidade no decorrer das sete semanas subseqüentes de treinamento.

Embora a amostra do presente estudo tenha sido composta por mulheres normotensas, uma redução significante nos valores de PAS, PAD e PAM, na ordem de 5, 8 e $6 \mathrm{mmHg}$, respectivamente, foi verificada após 16 semanas de TP $(P<0,05)$. Vale destacar que as reduções na PAS e PAM, aliadas a uma tendência de redução na PAD, já haviam sido encontradas nas primeiras oito semanas do presente estudo e, portanto, coincidem com o padrão temporal de remodelamento arterial periférico relatado por Rakobowchuk et al. ${ }^{(26)}$.

Apesar dos valores de queda da PA neste estudo serem superiores aos relatados pela maioria das investigações anteriores com TP (aproximadamente $3,2 \mathrm{mmHg}$ na PAS e $3,5 \mathrm{mmHg}$ na PAD)(15), reduções superiores às verificadas no presente estudo já haviam sido relatadas anteriormente por Carter et al.(24), em pesquisa realizada com jovens normotensos, de ambos os sexos, após oito semanas de TP $(9,8$ e $8 \mathrm{mmHg}$ para PAS, PAD e PAM, respectivamente). Vale ressaltar que a intensidade e o volume de treinamento empregado ( $3 \times 10-\mathrm{RM})$ foram relativamente semelhantes aos utilizados na presente investigação.

Por outro lado, Byrne e Wilmore ${ }^{(23)}$, após submeterem um grupo de mulheres normotensas a um programa de 20 semanas de TP, encontraram reduções em valores absolutos para a PAS ($3 \mathrm{mmHg})$, PAD $(-2 \mathrm{mmHg})$ e PAM $(-2 \mathrm{mmHg})$, apesar dessas modificações não terem sido confirmadas estatisticamente. Nesse estudo a intensidade e o volume de cada sessão de treinamento foram similares aos utilizados na presente investigação, contudo os autores empregaram uma freqüência semanal maior (quatro sessões), adotando um parcelamento (divisão) nas programações de treinamento, de modo que cada grupamento muscular foi estimulado em apenas duas sessões semanais. Embora o efeito isolado dessa variável (número de sessões) sobre o comportamento da PA em repouso ainda necessite ser investigado mais criteriosamente, acredita-se que a falta de similaridade no número de estimulações semanais, possa exercer algum impacto nas respostas pressóricas ao TP.

Com base nos achados disponíveis na literatura parece que as diferenças de magnitude nas respostas hipotensoras crônicas acarretadas pelo TP podem estar relacionadas, pelo menos em parte, às características da amostra estudada, bem como a manipulação isolada ou combinada de diversas variáveis que compõem os programas de treinamento utilizados (volume e intensidade de esforço, tempo de recuperação entre séries, número de exercícios, grupamentos musculares exercitados, forma de entre outras)(27).

Nesse sentido, o presente estudo utilizou duas formas de estruturação do programa de TP, na tentativa de oferecer uma progressão adequada de modo que as possíveis adaptações também fossem progressivas. Na primeira etapa (primeiras oito semanas) o programa de TP foi estruturado de acordo com uma montagem alternada por segmento, com os exercícios sendo executados em ordem semelhante à utilizada em TP em forma de circuito. Posteriormente, na segunda etapa (últimas oito semanas) o programa de TP foi reestruturado, de modo que os exercícios foram agrupados de acordo com os respectivos grupos musculares a serem treinados e foram executados segundo uma ordem predeterminada (dos grandes para os pequenos grupos musculares), diferenciando-se membros superiores e inferiores. Os resultados indicaram que as principais alterações na PAS e na PAM foram verificadas na primeira etapa, com estabilização dessas variáveis no decorrer da segunda etapa. Por outro lado, as melhores respostas da PAD foram identificadas somente após o término na segunda etapa.

Portanto, os achados do presente estudo sugerem que a forma de estruturação dos programas de TP pode acarretar respostas pressóricas diferenciadas. Não se pode descartar que a duração do protocolo também pode ter afetado a magnitude das respostas encontradas, além do que o delineamento adotado não permite analisar se o possível efeito da progressão adotada não teria comprometido, pelo menos em parte, tais respostas. Assim, seria interessante analisar, também, o impacto do programa estruturado na primeira etapa deste experimento por períodos mais prolongados ou, ainda, a adoção do programa estruturado na segunda etapa, já nas fases iniciais de treinamento.

Apesar dos mecanismos responsáveis pelas respostas hipotensoras crônicas acarretadas pelo TP ainda não estarem totalmente esclarecidos, acredita-se que reduções no débito cardíaco e na resistência vascular periférica total, possam explicar pelo menos em parte tais modificações, visto que após o esforço físico parece ocorrer um aumento mais acentuado de sustâncias vasodilatadoras na circulação, como é o caso do óxido nítrico(19). Por outro lado, especula-se que tais reduções possam ser causadas pela diminuição da atividade nervosa simpática, hipótese que tem sido refutada por alguns pesquisadores ${ }^{(24)}$. Infelizmente, tais hipóteses não foram testadas neste estudo.

Considerando que programas de TP podem proporcionar maiores reduções na PA em indivíduos hipertensos do que em normotensos ${ }^{(23)}$ e que uma redução de $5 \mathrm{mmHg}$ nos valores de PA pode reduzir o risco de desenvolvimento de doenças cardiovasculares de $40 \%$ para $15 \%$ em hipertensos ${ }^{(24)}$, os resultados encontrados neste estudo parecem extremamente promissores. Vale destacar que ainda existe a necessidade de confirmação de tais informações em mulheres hipertensas.

Embora o presente estudo apresente achados relevantes, não se pode desprezar o possível impacto da falta de controle dos hábitos nutricionais, bem como do número reduzido de sujeitos investigados.

\section{CONCLUSÃO}

Os resultados do presente estudo sugerem que 16 semanas de TP, realizado com freqüência de três sessões semanais, pode proporcionar importantes reduções na PA em mulheres jovens, normotensas e, previamente, não-treinadas.

\section{AGRADECIMENTOS}

Os autores agradecem o CNPq pelo auxílio financeiro e as bolsas outorgadas

Todos os autores declararam não haver qualquer potencial conflito de interesses referente a este artigo.

\section{REFERÊNCIAS}

1. Cooper RS, Wolf-Maier K, Luke A, Adeyemo A, Banegas JR, Forrester T, et al. An international comparative study of blood pressure in populations of European vs. African descent. BMC Med. [electronic resource] 2005;3

2. Carneiro G, Faria AN, Ribeiro FF, Guimarães A, Lerário D, Ferreira SRG, et al. Influência da distribuição da gordura corporal sobre a prevalência de hipertensão arterial e outros fatores de riscos em indivíduos obesos. Rev Ass Med Brasil. 2003;49:306-11.

3. Feijão AMM, Gadilha FV, Bezerra AA, Oliveira AM, Silva MSS, Lima JWO. Prevalência de excesso de peso e hipertensão arterial em população urbana de baixa renda. Arq Bras Cardiol. 2005;84:29-33.

4. Hagberg MJ, Park JJ, Brown MD. The role of exercise training in treatment of hypertension. Sports Med. 2000;30:193-206.

5. Farinatti PTV, Assis BFCB. Estudo da freqüência cardíaca, pressão arterial e duplo produto em exercícios contra-resistência e aeróbio contínuo. Rev Bras Ativ Fís Saúde. 2000;5:5-16.

6. Farinatti PTV, Oliveira RB, Pinto VLM, Monteiro WD, Francischetti E. Programa domiciliar de exercícios: efeitos de curto prazo sobre a aptidão física e pressão arterial de indivíduos hipertensos. Arq Bras Cardiol. 2005;84:473-9.

7. Whelton SP, Chin A, Xin X, He J. Effect of aerobic exercise on blood pressure: a meta-analyze of randomized, controlled trials. Ann Intern Med. 2002;136:493-503. 
8. Forjaz CLM, Matsudaira Y, Rodrigues FB, Nunes N, Negrão CE. Post-exercise changes in blood pressure, heart rate and rate pressure product at different exercise intensities in normotensive humans. Braz J Med Biol Res. 1998;31:1 247-55.

9. Forjaz CLM, Cardoso Jr CG, Rezk CC, Santaella DF, Tinucci T. Post-exercise hypotension and hemodynamics: the role of exercise intensity. J Sports Med Phys Fitness. 2004;44:54-62.

10. Hagberg JM, Graves JE, Limacher M, Woods DR, Leggertt SH, Cononie C, et al. Cardiovascular responses of 70- to 79-yr-old men and women to exercise training. J Appl Physiol. 1989;66:2589-94.

11. Schwartz RS, Hirth VA. The effects of endurance and resistance training on blood pressure. Int J Obes Relat Met Disord. 1995;19:S52-7.

12. Sociedade Brasileira de Hipertensão, Sociedade Brasileira de Cardiologia, Sociedade Brasileira de Nefrologia. IV Diretrizes Brasileiras de Hipertensão Arterial. Hipertensão. 2002;4:126-63.

13. Kelley GA. Dynamic resistance exercise and resting blood pressure in adults: a meta-analysis. J Appl Physiol. 1997;82:1559-65.

14. Kelley GA, Kelley KS. Progressive resistance exercise and resting blood pressure. A meta-analysis of randomized controlled trials. Hypertension. 2000;35: 83843.

15. Cornelissen VA, Fagard $\mathrm{RH}$. Effect of resistance training on resting blood pressure: a meta-analysis of randomized controlled trials. J Hypertension. 2005;23: 251-9.

16. American Association of Cardiovascular and Pulmonary Rehabilitation. Guidelines for cardiac rehabilitation and secondary prevention programs. $3^{\text {nd }}$ ed. Champaign: Human Kinetics Books; 1999.

17. American College of Sports Medicine. Position stand: the recommended quantity and quality of exercise for developing and maintaining cardiorespiratory and muscular fitness, and flexibility in healthy adults. Med Sci Sports Exerc. 1998; 30:975-91.

18. American College of Sports Medicine. Exercise and physical activity for older adults. Med Sci Sports Exerc. 1998;30:992-1008.

19. American College of Sports Medicine. Position stand: exercise and hypertension. Med Sci Sports Exerc. 2004;36:533-53.

20. Fletcher GF, Balady G, Froelicher VF, Hartley LH, Haskell WL, Pollock ML. Exercise standards: a statement for healthcare professionals from the American Heart Association. Circulation. 1995;91:580-615.

21. Gordon CC, Chumlea WC, Roche AF. Stature, recumbent length, and weight. In: Lohman TG, Roche AF, Martorell R, editors. Anthropometric standardizing reference manual. Champaign: Human Kinetics Books; 1988. p. 3-8.

22. Rodrigues CEC, Rocha PECP. Musculação: teoria e prática. Rio de Janeiro: Sprint; 1985.

23. Byrne HK, Wilmore JH. The effects of resistance training on resting blood pressure in women. J Strength Cond Res. 2000;14:411-8.

24. Carter JR, Ray CA, Downs EM, Cooke WH. Strength training reduces arterial blood pressure but not sympathetic neural activity in young normotensive subjects. J Appl Physiol. 2003;94:2212-6.

25. Forjaz CLM, Rezk CC, Melo CM, Santos DA, Teixeira L, Nery SS, et al. Exercício resistido para o paciente hipertenso: indicação ou contra-indicação. Rev Bras Hipertens. 2003;10:119-24.

26. Rakobowchuk M, McGowan CL, de Groot PC, Hartman JW, Phillips SM, MacDonald MJ. Endothelial function of young healthy males following whole body resistance training. J Appl Physiol. 2005;98:2185-90.

27. Fisher MM. The effects of resistance exercise on recovery blood pressure in normotensive and borderline hypertensive women. J Strength Cond Res. 2001; 15:210-6. 